PREPARED FOR THE U.S. DEPARTMENT OF ENERGY, UNDER CONTRACT DE-AC02-76CH03073

PPPL-3574

PPPL-3574

UC-70

Transient Transport Experiments in the CDX-U Spherical Torus

T. Munsat, P.C. Efthimion, B. Jones, R. Kaita, R. Majeski,

D. Stutman and G. Taylor

June 2001

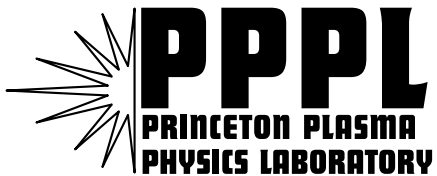

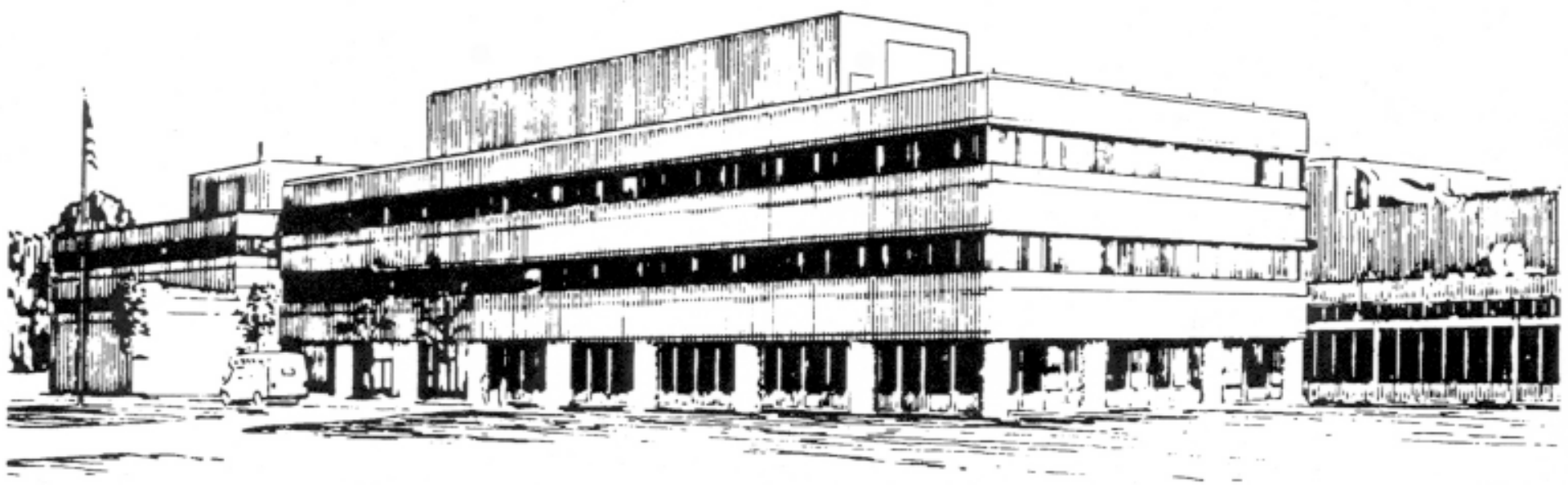

PRINCETON PLASMA PHYSICS LABORATORY PRINCETON UNIVERSITY, PRINCETON, NEW JERSEY 


\section{PPPL Reports Disclaimer}

This report was prepared as an account of work sponsored by an agency of the United States Government. Neither the United States Government nor any agency thereof, nor any of their employees, makes any warranty, express or implied, or assumes any legal liability or responsibility for the accuracy, completeness, or usefulness of any information, apparatus, product, or process disclosed, or represents that its use would not infringe privately owned rights. Reference herein to any specific commercial product, process, or service by trade name, trademark, manufacturer, or otherwise, does not necessarily constitute or imply its endorsement, recommendation, or favoring by the United States Government or any agency thereof. The views and opinions of authors expressed herein do not necessarily state or reflect those of the United States Government or any agency thereof.

\section{Availability}

This report is posted on the U.S. Department of Energy's Princeton Plasma Physics Laboratory Publications and Reports web site in Calendar Year 2001. The home page for PPPL Reports and Publications is: http://www.pppl.gov/pub_report/

DOE and DOE Contractors can obtain copies of this report from:

U.S. Department of Energy

Office of Scientific and Technical Information

DOE Technical Information Services (DTIS)

P.O. Box 62

Oak Ridge, TN 37831

Telephone: (865) 576-8401

Fax: (865) 576-5728

Email: reports@adonis.osti.gov

This report is available to the general public from:

National Technical Information Service

U.S. Department of Commerce

5285 Port Royal Road

Springfield, VA 22161

Telephone: 1-800-553-6847 or

(703) 605-6000

Fax: (703) 321-8547

Internet: http://www.ntis.gov/ordering.htm 


\title{
Transient Transport Experiments in the CDX-U Spherical Torus
}

\author{
T. Munsat, P.C. Efthimion, B. Jones, R. Kaita, R. Majeski, D. Stutman and G. Taylor \\ Plasma Physics Laboratory, Princeton University, Princeton, NJ 08543
}

(June 4, 2001)

\begin{abstract}
Electron transport has been measured in CDX-U using two separate perturbative techniques. Gas modulation at the plasma edge was used to introduce cold-pulses which propagate towards the plasma center, providing time-offlight information leading to a determination of $\chi_{\mathrm{e}}$ as a function of radius. Sawteeth at the $\mathrm{q}=1$ radius $(\mathrm{r} / \mathrm{a} \sim 0.15)$ induced heat-pulses which propagated outward towards the plasma edge, providing a complementary time-of-flight based $\chi_{\mathrm{e}}$ profile measurement. This work represents the first localized measurement of $\chi_{\mathrm{e}}$ in a spherical torus. It is found that $\chi_{\mathrm{e}}=1-2 \mathrm{~m}^{2} / \mathrm{s}$ in the plasma core $(\mathrm{r} / \mathrm{a}<1 / 3)$, increasing by an order of magnitude or more outside of this region. Furthermore, the $\chi_{\mathrm{e}}$ profile exhibits a sharp transition near $\mathrm{r} / \mathrm{a}=1 / 3$. Spectral and profile analyses of the soft x-rays, scanning interferometer, and edge probe data show no evidence of a significant magnetic island causing the high $\chi_{\mathrm{e}}$ region.
\end{abstract}

Typeset using REVTEX 


\section{INTRODUCTION}

In recent years there has been increased interest in the spherical torus (ST) geometry as a means of obtaining high fusion power density at relatively modest magnetic field $[1,2]$. Ideal MHD studies have found stable equilibria in this configuration featuring $\beta \sim 50 \%$ and pressure-driven bootstrap current fractions of $f_{B S} \sim 100 \%$ [3-5], and the START ST has achieved $\beta \sim 30 \%$ in the laboratory [6,7]. Additionally, certain MHD instabilities have been predicted to stablize as the aspect ratio $(\mathrm{R} / \mathrm{a})$ falls below $1.5[8,2]$.

Confinement predictions for the ST geometry have been more sparse, though, with most studies limited to the application of global confinement scalings originally developed for conventional tokamaks $[9,10]$. This will change in the coming years, with the introduction of the large ST experiments NSTX (PPPL) and MAST (Culham). Transport studies are of utmost importance to the development of the ST as a candidate fusion reactor, since many

of the most promising MHD phenomena depend on highly tailored pressure and current profiles.

The entire collection of thermal transport data in ST's up to now can be covered by summarizing the results from the START device $\left(\mathrm{I}_{\mathrm{P}} \sim 200 \mathrm{kA}, \mathrm{B}_{\mathrm{T}} \sim 4-6 \mathrm{kG}, \mathrm{T}_{\mathrm{e}} \sim 300-500\right.$ $\mathrm{eV}, \mathrm{R} / \mathrm{a}=1.3-1.5)$ [11], including both ohmic and NBI-heated plasmas. Global confinement studies of ohmic START plasmas have shown $\tau_{\mathrm{E}} \sim 0.5-1.2 \mathrm{~ms}$ [12], with reasonable agreement found with the semi-empirical modified Lackner-Gottardi model [13,14]. Calculation of $\chi_{\mathrm{e}}$ profiles based on power-balance considerations were performed for high-performance NBI-heated START discharges, resulting in estimates of $\chi_{\mathrm{e}} \sim 20 \mathrm{~m}^{2} / \mathrm{s}$ and $\chi_{\mathrm{i}} \sim 5-10 \mathrm{~m}^{2} / \mathrm{s}$ [7]. Again a reasonable agreement was found between the magnitude of $\chi_{\mathrm{e}}$ and LacknerGottardi estimates, though the profile shape showed a poor match to the model. $\chi_{i}$ was found to be in reasonable agreement with Chang-Hinton neoclassical estimates. Neoclassical $\chi_{\mathrm{e}}$ estimates were not performed.

In the present experiment, perturbative techniques are used in the CDX-U ST $\left(\mathrm{I}_{\mathrm{P}} \sim 70\right.$ $\left.\mathrm{kA}, \mathrm{B}_{\mathrm{T}} \sim 2.3 \mathrm{kG}, \mathrm{T}_{\mathrm{e}} \sim 100 \mathrm{eV}, \mathrm{R}_{0}=34 \mathrm{~cm}, \mathrm{a}=22 \mathrm{~cm}, \mathrm{~A}=\mathrm{R}_{0} / \mathrm{a}=1.5\right)$ to derive localized 
measurements of the $\chi_{\mathrm{e}}$ profile.

Perturbative transport experiments have been performed on nearly all major fusion devices over the past decade [15-24], and can offer several advantages over steady-state techniques. These include decoupling of density and temperature gradients from each other, and a decoupling of temperature and density from their gradients, with no essential degradation of the quality of the data [25].

One feature of perturbative analysis that is particularly relevant to the CDX-U experiment is that of diagnostic instrumentation. For power-balance analysis, one is required to know with great certainty the profiles of electron temperature, ion temperature, electron and ion density, and all power sources and sinks, including ohmic heating and radiative losses. Though it is not necessary to know the time dependence of these quantities, all quantities must be known in order to calculate either of $\chi_{\mathrm{e}}$ or $\chi_{\mathrm{i}}$. Transient techniques, by contrast, are critically dependent on time resolved data, but are only weakly dependent on absolute calibration and require only a subset of the above measurements to measure either $\chi_{\mathrm{e}}$ or $\chi_{\mathrm{i}}$. Furthermore, the time-dependent analysis of plasma perturbations is performed when heat and particle sources and sinks can be ignored. This type of analysis is well suited to CDX-U, which lacks $T_{i}$ diagnostics and has only an uncalibrated estimate of radiated power, but is equipped with spatially and temporally resolved $\mathrm{T}_{\mathrm{e}}$ diagnostics. The EBW $\mathrm{T}_{\mathrm{e}}$ diagnostic, while not absolutely calibrated, provides time-resolved profiles of radiation temperature, and the SXR array provides time-resolved CV emission profiles.

Historically, $\chi_{\mathrm{e}}$ values derived from perturbative techniques have exceeded $\chi_{\mathrm{e}}$ derived from power balance by a factor of 1-5 or more. Nonlinear gradient dependence or fast nonlocal transport are typically cited as explanations for this discrepancy, though recent work has demonstrated that transient MHD activity may dominate these effects by introducing weak stochasticity to the local field structure [26]. 


\section{HEAT-PULSE EXPERIMENTS USING SAWTEETH PERTURBATIONS}

Electron thermal transport in CDX-U was assessed by recording the spatial and temporal evolution of sawtooth-induced perturbations with the soft x-ray (SXR) array [27]. A layout of the array, including the intersection of the viewing chords with the CDX-U flux-surfaces, is shown in Fig. 1. The $\mathrm{r}$ value for each viewing chord was calculated by taking the outermost flux surface intersected by the chord and mapping it back to the midplane, assuming that thermal transport along a magnetic surface is much faster than transport across compressed magnetic surfaces. The array is vertically symmetric about the midplane, so that the 30 viewing chords correspond to 15 radial observation locations: $\mathrm{r}_{\mathrm{obs}}[\mathrm{cm}]=13.9,13.0,12.1$, $11.2,10.3,9.4,8.4,7.5,6.5,5.5,4.5,3.5,2.4,1.4,0.3$.

The spatio-temporal evolution of a diffusion-dominated temperature perturbation is governed by the perturbed electron heat equation

$$
\frac{3}{2} n_{e} \frac{\partial \tilde{T}_{e}(r, t)}{\partial t}=\frac{1}{r} \frac{\partial}{\partial r}\left[r n_{e} \chi_{e} \frac{\partial \tilde{T}_{e}(r, t)}{\partial r}\right]
$$

In the case of sawteeth-induced perturbations, the initial conditions for this equation can be taken as a pair of opposing $\delta$-functions located at the inversion radius. An analytical solution to this equation with $\delta$-function initial conditions was obtained in [28] and is given by

$$
\begin{aligned}
\tilde{T}_{e}(r, t)= & \frac{A^{2} r_{1} r_{s} \tilde{T}_{e 0}}{2 t} \exp \left(\frac{-A^{2} r^{2}}{4 t}\right) \times \\
& {\left[\exp \left(\frac{-A^{2} r_{2}^{2}}{4 t}\right) \mathrm{I}_{0}\left(\frac{\mathrm{A}^{2} \mathrm{rr}_{2}}{2 \mathrm{t}}\right)-\exp \left(\frac{-\mathrm{A}^{2} \mathrm{r}_{1}^{2}}{4 \mathrm{t}}\right) \mathrm{I}_{0}\left(\frac{\mathrm{A}^{2} \mathrm{rr}_{1}}{2 \mathrm{t}}\right)\right] }
\end{aligned}
$$

where $t$ is the time measured from the sawtooth time $t_{s}, r_{s}$ is the sawtooth inversion radius, $\mathrm{r}_{0}\left(\leq \sqrt{2} \mathrm{r}_{\mathrm{s}}\right)$ is the reconnection radius, and $\mathrm{r}_{1}, \mathrm{r}_{2}$ are the positions of the negative and positive delta functions, respectively (each of magnitude $\tilde{\mathrm{T}}_{\mathrm{e} 0}$ ), and $\mathrm{A}=\sqrt{1.5 / \chi_{\mathrm{e}}}$.

An approximate solution to this equation was derived in [15] which leads to a description of the perturbation trajectory given by

$$
\Delta t_{\text {peak }} \approx \frac{\left(\Delta r_{\text {peak }}\right)^{2}}{13.5 A^{2}}=\frac{\left(\Delta r_{\text {peak }}\right)^{2}}{9 \chi_{e}}
$$


where $\Delta t_{\text {peak }}$ is the delay between the initial perturbation and the local measurement, and $\Delta r_{\text {peak }}$ is the difference between the location of the local peak and the location of the initial perturbation.

In the CDX-U experiments, the raw SXR data was best-fit to the evolved perturbation function given by equation 2.2, parameterized by $\chi_{\mathrm{e}}, \mathrm{t}_{\mathrm{s}}, \mathrm{r}_{\mathrm{obs}}$, and a scalar magnitude. $\mathrm{t}_{\mathrm{s}}$ and $r_{\mathrm{obs}}$ were fixed according to the location of the observed sawtooth, and $\chi_{\mathrm{e}}$ was derived by finding a best-fit to the data at each measurement chord. A series of SXR waveforms covering the full range of the SXR array outside the sawtooth radius, plotted with the best-fit solutions of equation 2.2 is shown in Fig. 2.

It is important to notice the progression from one channel to the next as the pulse travels from the $\mathrm{q}=1$ surface outward. Channels at $\left(\mathrm{r}-\mathrm{r}_{\mathrm{s}}\right)<5 \mathrm{~cm}$ demonstrate a pulse evolution well represented by equation 2.2 with $\chi_{\mathrm{e}}=1.1 \mathrm{~m}^{2} / \mathrm{s}$. While the $\Delta r$ covered between Fig. $2 \mathrm{a}$ and $2 \mathrm{~d}$ is $3.9 \mathrm{~cm}$, nearly identical to that covered between Fig. $2 \mathrm{~d}$ and $2 \mathrm{e}(3.6 \mathrm{~cm})$, the pulse shape undergoes significant relaxation/delay over the first $3.9 \mathrm{~cm}$, and almost no further evolution over the last $3.6 \mathrm{~cm}$, indicating relatively rapid thermal transport through this outer region.

In order to illustrate this behavior more precisely, the $\Delta t_{\text {peak }}$ values for each channel are plotted against the observation radius for that channel, producing a map of the pulse trajectory in space and time, shown in Fig. 3a.

The quadratic fit representing $\chi_{\mathrm{e}}=1.1 \mathrm{~m}^{2} / \mathrm{s}$ (from eq. 2.3) accurately represents the heat pulse delay data only for $r_{s}<r<10.5 \mathrm{~cm}$, while $\chi_{\mathrm{e}}$ is seen to increase sharply outside of this region.

Although it is difficult to assess a $\chi_{\mathrm{e}}$ value in the outer region with reasonable precision, it appears to exceed the core value by an order of magnitude or more. The analysis of several similar sawtoothing discharges showed very uniform profile behavior, with derived $\chi_{\mathrm{e}}$ values in the core ranging from 0.8 to $2.2 \mathrm{~m}^{2} / \mathrm{s}$.

Figures $3 \mathrm{~b}$ and $3 \mathrm{c}$ show $\mathrm{T}_{\mathrm{e}}$ and $\mathrm{n}_{\mathrm{e}}$ profiles from TVTS, supplemented by an edge measurement $(\mathrm{r}=22 \mathrm{~cm})$ from a triple Langmuir probe. The steepest temperature gradient 
exists in the region of measured low $\chi_{\mathrm{e}}(\mathrm{r} / \mathrm{a} \sim 1 / 3)$, and a much flatter (and colder) region exists where $\chi_{\mathrm{e}}$ is measured to be sharply higher.

The $n_{e}$ profile (fig. 3c) also exhibits a steep central gradient and peak, though the $n_{e}$ gradient appears at smaller radius than the temperature gradient. The $\mathrm{n}_{\mathrm{e}}$ peak, as well as the corresponding dip in $\mathrm{T}_{\mathrm{e}}$, appear within the $\mathrm{q}=1$ radius, as indicated in the figure.

\section{COLD-PULSE EXPERIMENTS USING EDGE GAS-PUFFING}

A complementary $\chi_{\mathrm{e}}$ measurement was performed using similar time-to-peak methods applied to cold-pulses propagating inward from the plasma edge. For these experiments, gas-puffing modulations were introduced to a background plasma which acted to locally cool the edge, and the temperature response was measured using the electron Bernstein wave (EBW) $\mathrm{T}_{\mathrm{e}}$ diagnostic [29]. The $\mathrm{T}_{\mathrm{e}}$ perturbation was measured at the plasma edge with a triple Langmuir probe. A series of reproducible discharges was taken with the EBW radiometer scanning in frequency either shot-to-shot or many times within a single shot. The EBW frequency range was 8.4-12.4 GHz, corresponding to second-harmonic EBW emission covering $\mathrm{R}=35.7-53.3 \mathrm{~cm}$, roughly the center to the outer edge of the plasma. The triple probe was held fixed at the plasma edge $(R=56 \mathrm{~cm})$ for all shots.

For experiments using the fast-scanning radiometer, the frequency was swept at 50 frequency sweeps per millisecond. Data was sampled at $1 \mathrm{MHz}$, providing 20 points per sweep, though the phase of the frequency sweep was uncorrelated with the phase of the sampling. EBW emission intensity vs. time and frequency was interpolated over an irregular grid, and data from several identical shots were summed to improve signal-to-noise levels. The combined data was then regridded to provide a set of time traces at fixed frequency locations (each corresponding to a radius in the plasma). The local minimum at the cold-pulse was then located for each radius. The resulting dataset is shown in Fig. 4.

Because of the regridding process, interpolated time traces were produced at several

hundred radius locations. In order to faithfully represent the original sampling rate (and 
not present falsely high resolution in the data points), the analyzed data was re-binned into 20 evenly spaced radial locations.

To model the edge perturbations, equation 2.1 was numerically solved using a timedependent boundary condition at the plasma edge corresponding to a negative temperature perturbation. The subsequent relaxation over the plasma profile was evolved in time, and from this the trajectory of the perturbation peak was plotted to provide a straightforward means of comparison to experiment. Plotted with the data is the result of the simulation over the ranges $\chi_{\mathrm{e}}=1.0-5.4 \mathrm{~m}^{2} / \mathrm{s}$ and $r_{\text {perturbation }}=5.3-9.9 \mathrm{~cm}$.

Error bars in major radius $(R)$ were produced by perturbing each of the quantities used to provide the mapping between frequency and major radius, namely $\mathrm{B}_{\mathrm{T}}$ and $\mathrm{I}_{\mathrm{P}}(\mathrm{r})$, by their estimated error, and also taking into account the error introduced by the finite bandwidth of the radiometer electronics. Each contribution to the overall error in $\mathrm{R}$ was then added in quadrature, though in practice the radiometer bandwidth dominated the other effects. Errors in time represent the scatter in the derived temperature peaks within each bin of overgridded time traces.

The reasonable fit between the data and model implies that thermal transport in the CDX-U core can be described by a diffusive process, with a diffusion coefficient of $\chi_{\mathrm{e}} \sim$ $3 \mathrm{~m}^{2} / \mathrm{s}$. An additional important result comes from the fact that the data is well modeled by a perturbation originating around $\mathrm{r}=7.5 \mathrm{~cm}$, corresponding to $\mathrm{r} / \mathrm{a} \sim 1 / 3$. Outside this region, the data points are quite flat, which implies either that the diffusive transport is very fast beyond $\mathrm{r}=7.5 \mathrm{~cm}$, or that the perturbation introduced by the cold-pulse is not diffusive outside the plasma core. This observation is similar to the sawtooth-induced heatpulse propagation results in section II

Similar coldpulse experiments were performed using a slightly different experimental arrangement, with similar results. In these sets, the EBW radiometer sampled a single frequency during each shot, and was scanned between shots over the range 8.4-12.4 GHz over the course of several similar shots. This provided a higher sampling rate at each frequency, but lacked single-shot profile information. In these sets, a record of the pulse 
at the plasma edge was recorded by either the triple Langmuir probe or by a second EBW radiometer at a fixed reference frequency corresponding to the plasma edge. Analysis of these datasets resulted in $\chi_{\mathrm{e}}=1.5-5.9 \mathrm{~m}^{2} / \mathrm{s}$ and $\mathrm{r}_{\text {perturbation }}=5.5-9.5 \mathrm{~cm}$, corresponding to $\mathrm{r}_{\text {perturbation }} / \mathrm{a}=0.25-0.43$. As in the previous set, the data fits the model reasonably well inside the core and flattens out beyond $\mathrm{r} / \mathrm{a} \sim 1 / 3$, indicating an increase in transport outside the core.

In experiments such as these in which the temperature is perturbed through fueling modification, it is critical to investigate the possibility of coupling between the density and temperature perturbations, and the effect that it will have on the measured diffusion coefficients. A mathematical outline of the coupling between density and temperature perturbations and the effect of off-diagonal elements in the transport matrix is given in [30]. The primary finding of this work is an analytical treatment of the effect that gradient-dependent transport coefficients will have on perturbative measurements as compared to steady-state methods. It is also found that coupling of the temperature and density perturbations can lead to a separate "fast" and "slow" eigenmode solution to the diffusion equation, with corresponding pulse propagation rates.

In the CDX-U experiments, separate propagation eigenmodes were not observed, though the quality of the data may not be sufficient to resolve subdominant pulses or to resolve the possible coupling between $n_{e}$ and $T_{e}$ pulses. In particular, the edge cold-pulse experiment is the most susceptible to coupling between $n_{e}$ and $T_{e}$ diffusion, since the $T_{e}$ perturbation is a direct consequence of an induced $n_{e}$ perturbation. Indeed, the $\chi_{\mathrm{e}}$ values from the edge coldpulses were consistently higher than those determined from the sawtooth analysis, consistent with a finite coupling effect.

In trying to determine the source of the sharp transition in transport behavior observed in CDX-U, it is important to consider the possibility that transport is locally enhanced by a large magnetic island. In CDX-U, evidence for magnetic islands is obtained with both the SXR array and the scanning microwave interferometer, both of which sample nearly the full plasma radius. Additional information may be provided by the triple probe, located at 
$\mathrm{r}=22 \mathrm{~cm}$, and the Mirnov coils at the vessel wall.

Representative spectra of the SXR signal fluctuations, taken at $\mathrm{r}=4.5 \mathrm{~cm}$ and 10.3 $\mathrm{cm}$ (chosen to best represent the low and high $\chi_{\mathrm{e}}$ regions while remaining above the noise floor), are shown in Fig. 5. The spectra have been normalized to the mean signal level for each channel. Also shown is the noise-floor imposed by amplifier noise and digitization bit-noise, calculated by using signals from the SXR array recorded before the plasma shot. (A photon-noise floor was also calculated, but is negligible compared to both the signal level in Fig. 5a or the bit-noise floor in Fig. 5b.)

Fluctuations at $\mathrm{r}=4.5 \mathrm{~cm}$ show the $\mathrm{q}=1$ island at $\sim 10-12 \mathrm{kHz}$ and harmonics. Fluctuations at $\mathrm{r}=10.3 \mathrm{~cm}$ exhibit nearly white noise, reaching a noise floor at $\sim 10^{-7}$. Importantly, the frequency band $f<20 \mathrm{kHz}$ is above the noise floor in both plots, and the relative fluctuation level in the high $\chi_{\mathrm{e}}$ region is equal to or lower than that in the low $\chi_{\mathrm{e}}$ region.

Similar analysis was performed on signals from a microwave interferometer, scanned (shot-to-shot) from the plasma center to the edge, as well as on signals from a triple Langmuir probe at the plasma edge (both sampled at $100 \mathrm{kHz}$ ). Neither diagnostic showed evidence for magnetic islands outside of the $\mathrm{q}=1$ surface, and the interferometer scan showed comparable fluctuation levels in the high and low confinement regions. Additionally, spectral analysis of the Mirnov-coil array at the vessel wall showed dominant $10 \mathrm{kHz}$ activity, corresponding to the $\mathrm{q}=1$ island.

The dominant MHD activity in CDX-U appears to be the $\mathrm{q}=1$ magnetic island, located well within the higher confinement region. Given the magnitude of this island and the size of the high $\chi_{\mathrm{e}}$ region, it would seem that any magnetic island capable of causing the observed transport transition would need to be quite significant, though the fluctuation analysis shows no evidence of a magnetic island in the vicinity of the $\chi_{\mathrm{e}}$ transition. 


\section{COMPARISON WITH TRANSPORT THEORY AND SCALING LAWS}

Although it is widely believed that microturbulence of one form or another is the dominant mechanism leading to measured transport coefficients, it is nonetheless useful to maintain a comparison of any experimental data with neoclassical estimates. In particular, it has been predicted that the ST geometry may lead to stabilization of low-n MHD modes $[8,2]$. Though tokamak experiments have yet to achieve neoclassical electron confinement,

both neoclassical ion thermal confinement [31,7] and ion particle confinement [32] have been observed. Additionally, core electron thermal transport barriers have been observed in both DIII-D and RTP with auxiliary electron heating, though even in these cases electron thermal transport remained anomalously high [33,34].

Neoclassical estimates of all transport coefficients have been well established for high aspect ratio geometry, often using inverse aspect-ratio as an expansion parameter in the derivation by Hirshman [35]. The commonly used Chang-Hinton expression for $\chi_{\mathrm{i}}$ which incorporates finite aspect-ratio effects and interpolates between different collisionality regimes is given by Chang, et al. [36]. Neoclassical estimates for $\chi_{\mathrm{e}}$ which include finite aspect-ratio effects and span all collisionality regimes have recently been developed by Houlberg [37], and incorporated into the NCLASS and FORCEBAL codes.

The solid line in Fig. 6, labeled $\chi_{\mathrm{FBAL}}$, shows the $\chi_{\mathrm{e}}$ estimates calculated by the FORCEBAL code, using the profiles shown in figure 3 and a magnetic equilibrium geometry produced by the EFIT code for these shots. The shaded region in Fig. 6 represents the full range of measured $\chi_{\mathrm{e}}$ values from the temperature pulse measurements. Though not shown in the figure, recall that the experiments also demonstrate a sharp increase in $\chi_{\mathrm{e}}$ for $\mathrm{r}>10 \mathrm{~cm}$.

Clearly $\chi_{\mathrm{FBAL}}$ is a very poor match to the measured $\chi_{\mathrm{e}}$, both in magnitude and profile shape. The measured $\chi_{\mathrm{e}}$ is 10 times higher than $\chi_{\mathrm{FBAL}}$ in the core, and the qualitative experimental evidence for increased edge transport bears no resemblance to the $\chi_{\text {FBAL }}$ profile.

Importantly, the temperature profile provided by the Thomson scattering (fig. 3b) has a fairly large error-bar at the outermost datapoint $(\mathrm{r}=6 \mathrm{~cm})$, which can propagate to the 
$\chi_{\text {FBAL }}$ estimate. To address this possibility, a range of temperature profiles representing spline fits to datapoints covering the error-bar range shown in Fig. 3b were used in FORCEBAL. The resulting $\chi_{\text {FBAL }}$ profiles showed no substantial difference to the profile shown in Fig. 6 .

Interestingly, if the Chang-Hinton expression for $\chi_{\mathrm{i}}$ is modified for electrons, by replacing all ion quantities $\left(\nu_{\mathrm{i}}, \rho_{\mathrm{i}}\right.$, etc.) with the corresponding electron quantities, the resulting $\chi_{\mathrm{e}}$ profile shape matches the CDX-U data somewhat more closely. The result of this calculation is shown in figure 6 , labeled $\chi_{\mathrm{MCH}}$, along with a second scaling, labeled $\chi_{\mathrm{LAG}}$ and described below. The modified Chang-Hinton expression $\chi_{\mathrm{MCH}}$ is 5 times lower than the measured CDX-U value, somewhat comparable to the result obtained using FORCEBAL. The profile is a closer match to the CDX-U data, though the $\chi_{\mathrm{MCH}}$ estimate places the $\chi_{\mathrm{e}}$ gradient at a slightly larger $\mathrm{r}$ value than that measured in CDX-U.

Also shown in Fig. 6 is the semi-empirical Lackner-Gottardi $\chi_{\mathrm{e}}$ model, labeled $\chi_{\mathrm{LAG}}$. This curve uses the form modified by Connor for low aspect-ratio [14,13], which has been used in past comparisons to START transport data. The $\chi_{\mathrm{LAG}}$ scaling is intended for ohmic and L-mode plasmas, based on a simple model in which trapped particles are displaced by a banana width on each bounce.

$\chi_{\mathrm{LAG}}$ reproduces the measured $\chi_{\mathrm{e}}$ profile very poorly, and also overestimates the minimum $\chi_{\mathrm{e}}$ by a factor of 40. Interestingly, this is the model that has produced the best agreement with START power-balance based $\chi_{\mathrm{e}}$ data, in magnitude if not always in profile shape. It should also be noted that in the START high-performance NBI-heated discharges, the $\chi_{\mathrm{LAG}}$ estimates were very close to those calculated for CDX-U, though in that case the $\chi_{\mathrm{LAG}}$ estimates were a much closer match to the experimental $\chi_{\mathrm{e}}$ values [7].

In general, the comparisons to the two neoclassical and two semi-empirical $\chi_{\mathrm{e}}$ estimates is quite unsatisfactory. Only one model (modified Chang-Hinton expression) seems to vaguely represent the measured $\chi_{\mathrm{e}}$ behavior, and even this is a factor of five too low. Interestingly, the models chosen both underestimate and overestimate the measured $\chi_{\mathrm{e}}$. With the estimates covering such a wide range of predictions, it is difficult to draw concrete conclusions from 
the comparisons.

\section{CONCLUSIONS}

The electron transport profile has been measured in CDX-U using two independent perturbative methods, representing the first local measurements of $\chi_{\mathrm{e}}$ in a spherical torus. $\chi_{\mathrm{e}}$ is found to be $1-2 \mathrm{~m}^{2} / \mathrm{s}$ in the CDX-U core $(\mathrm{r} / \mathrm{a} \leq 1 / 3)$, sharply increasing outside of this region by an order of magnitude or more. $\mathrm{T}_{\mathrm{e}}$ profiles exhibit steep gradients in the region of low measured $\chi_{\mathrm{e}}$, reaffirming the conclusion of distinct regions of high and low transport levels. It appears from all available diagnostics that the profile behavior is not caused by a degradation of confinement due to the presence of a magnetic island. Though $\mathrm{D}_{\mathrm{e}}$ was not measured explicitly in these experiments, the $n_{e}$ profiles also exhibit steep gradients in the core region, though at a measurably different radius than the thermal gradients. Specifically, the $n_{e}$ peak appears inside the $q=1$ radius, and the $T_{e}$ gradient region appears outside of $q=1$. The location of other rational $q$ surfaces has not been measured in these plasmas.

The measured $\chi_{\mathrm{e}}$ level is a factor of 5-10 higher than neoclassical estimates, with a poor match to the profile shape. Likewise, two semi-empirical $\chi_{\mathrm{e}}$ scalings which have shown somewhat favorable comparison to START electron transport data show poor agreement with the CDX-U experimental results.

Several studies have been performed on other tokamaks which indicate that $\chi_{\mathrm{e}}$ measured by perturbative techniques can be several times higher than that determined by powerbalance techniques, due to nonlinearities in the dependence of $\chi$ on $\nabla \mathrm{T}$, local MHD perturbations, or coupled $n_{\mathrm{e}}$ and $\mathrm{T}_{\mathrm{e}}$ diffusion. Comparison with neoclassical $\chi_{\mathrm{e}}$ calculations indicate that the experimental values in CDX-U are 5-10 times higher than neoclassical estimates, though the steady-state experimental value may be closer than this. 


\section{FIGURES}

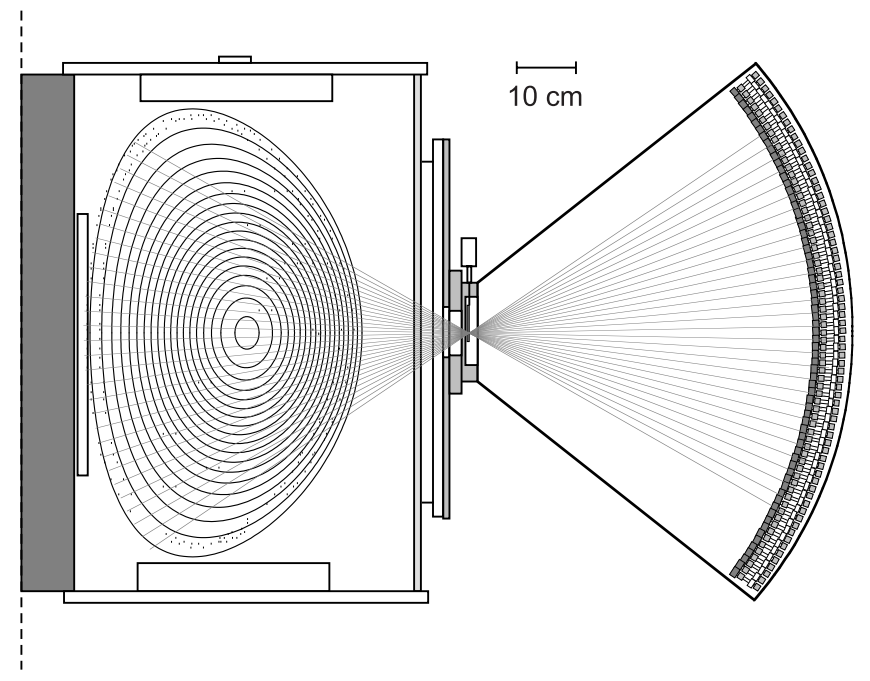

FIG. 1. Layout of SXR array, including viewing chords and CDX-U flux surfaces. 

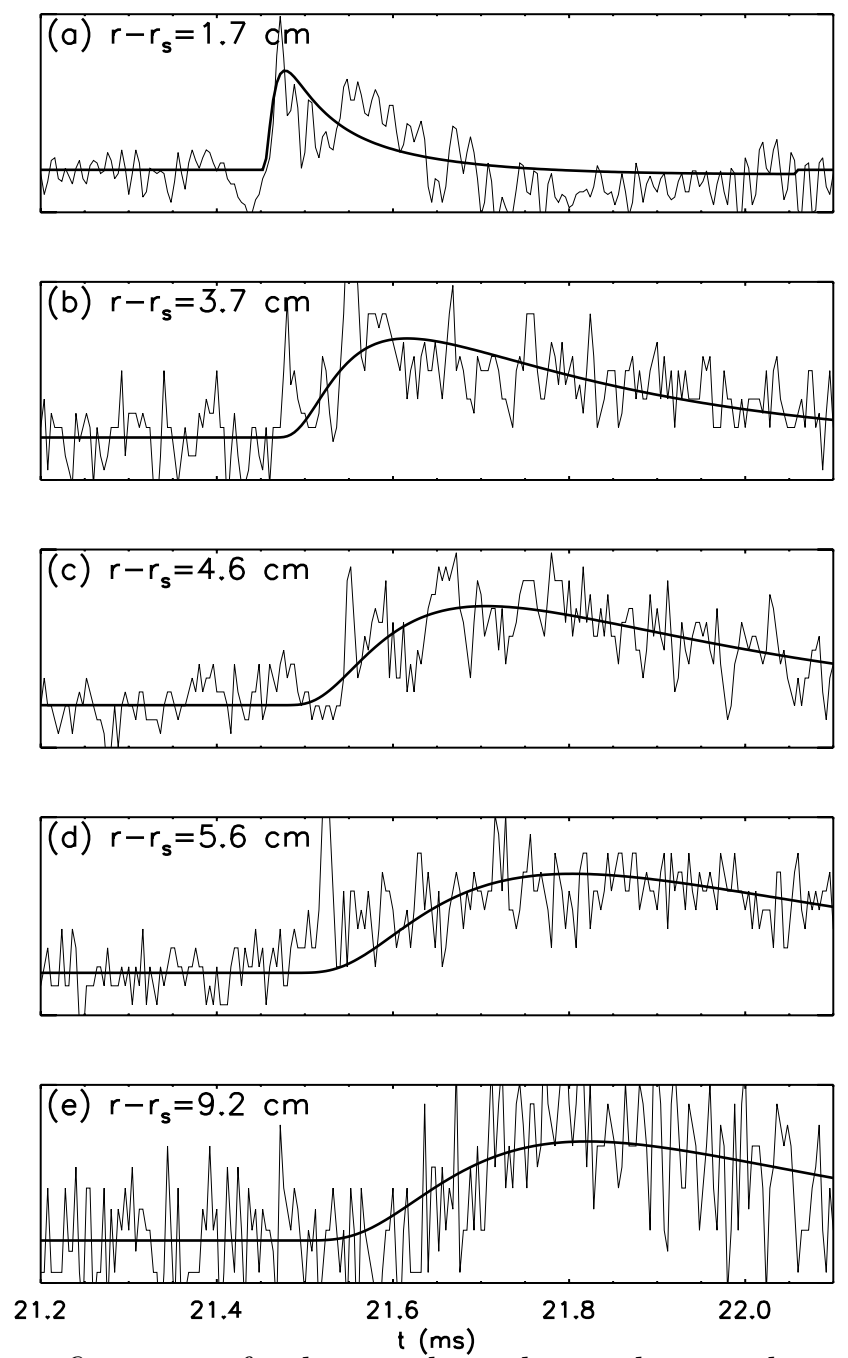

FIG. 2. Data and best-fit curves for heat-pulses observed around $t=t_{s}$ and at $r>r_{s}$, shot 0721001256. The radial difference between plots (a) and (d) is nearly identical to that between plots (d) and (e), though the pulse shape evolves significantly in the former case, and is nearly identical in the latter. 

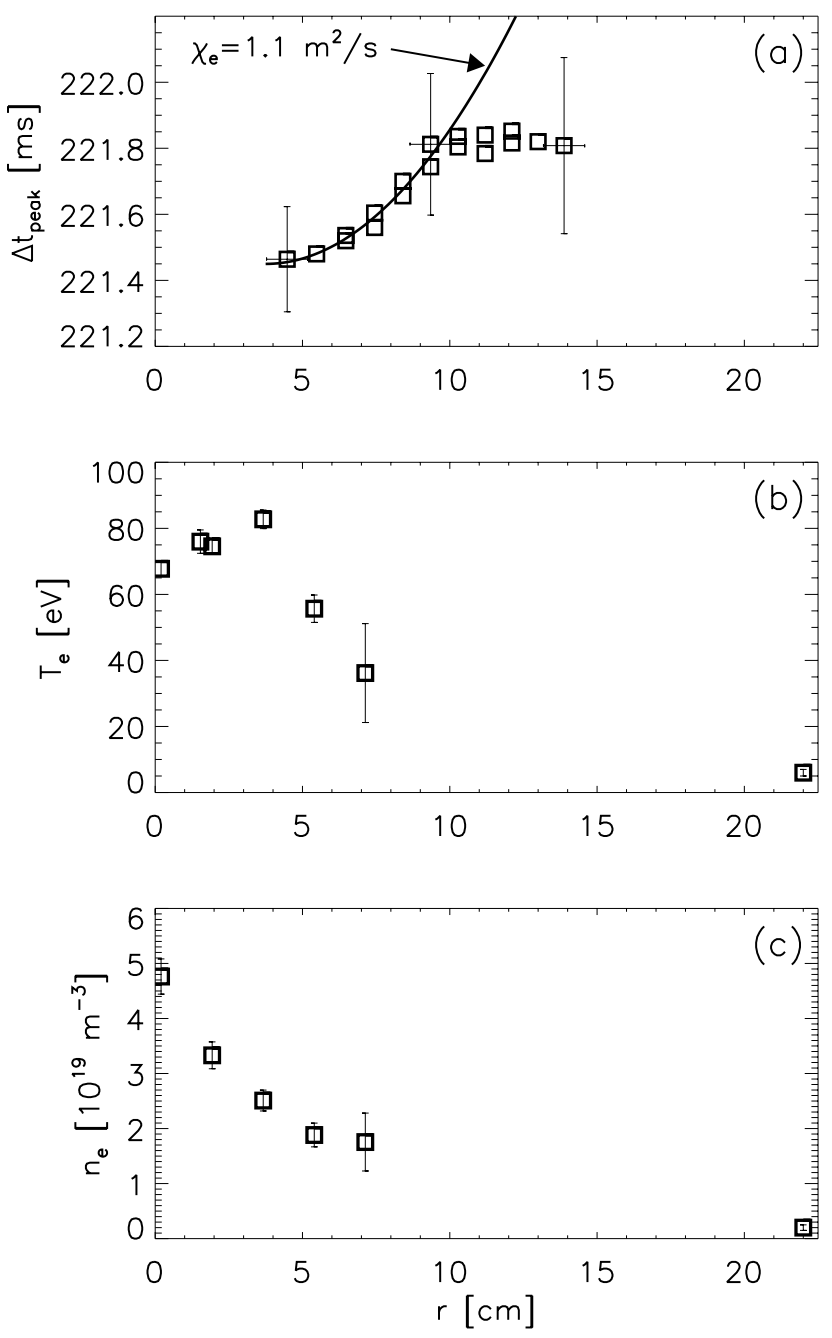

FIG. 3. Plot of heatpulse delay (a), electron $\mathrm{T}_{\mathrm{e}}(\mathrm{b})$ and $\mathrm{n}_{\mathrm{e}}(\mathrm{c})$ profiles versus minor radius. The heat-pulse trajectory is fit to a quadratic representing $\chi_{\mathrm{e}}=1.1 \mathrm{~m}^{2} / \mathrm{s}$ for $r_{s}<r<10.5 \mathrm{~cm}$. The steep $\mathrm{T}_{\mathrm{e}}$ gradient at $\mathrm{r} / \mathrm{a} \sim 1 / 3$ matches the region of inferred low $\chi_{\mathrm{e}}$. The $\mathrm{n}_{\mathrm{e}}$ profile is peaked within the $\mathrm{q}=1$ radius, as shown. 


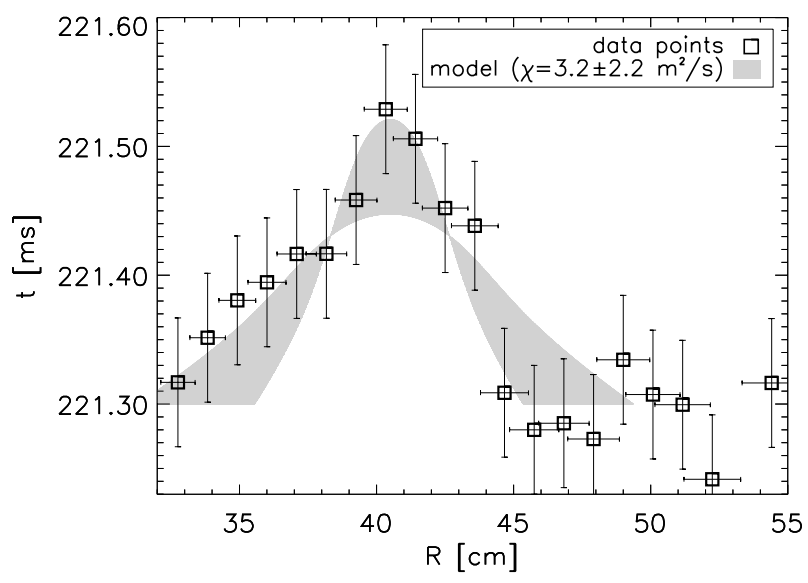

FIG. 4. Plot of heat pulse delay versus major radius, comparing $\chi_{\mathrm{e}}$ model to data from fast-scanning EBW radiometer. The shaded region corresponds to model parameters covering $\chi_{\mathrm{e}}=3.2 \pm 2.2 \mathrm{~m}^{2} / \mathrm{s}$ and $r_{\text {perturbation }}=7.6 \pm 3.3 \mathrm{~cm}$, i.e. a perturbation localized around $r_{\text {perturbation }} / a \sim 1 / 3$. 

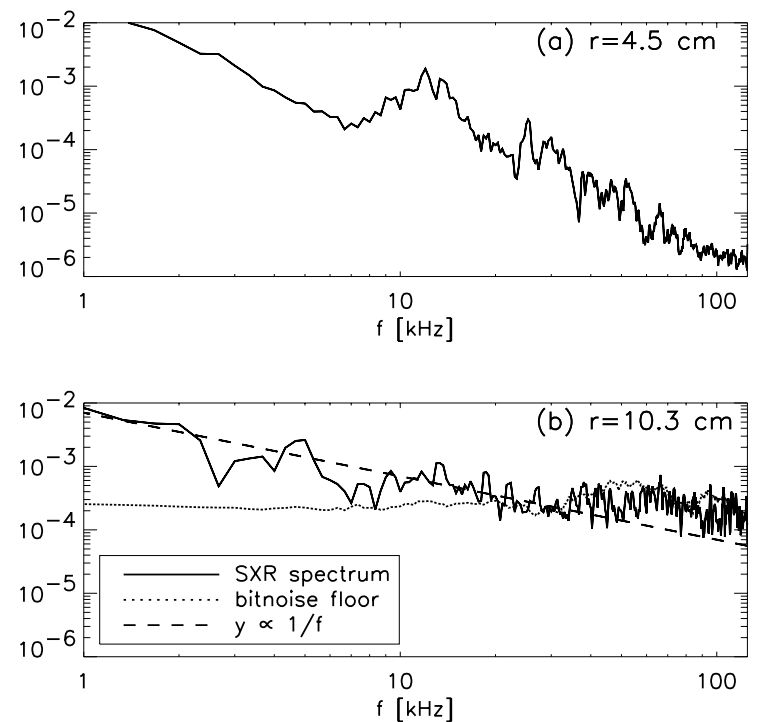

FIG. 5. Fluctuation spectra of the SXR signals, taken at two representative radii; (a) $\mathrm{r}=4.5$ $\mathrm{cm}$ and (b) $10.3 \mathrm{~cm}$ represent the low and high $\chi_{\mathrm{e}}$ regions, respectively. Fluctuations at $\mathrm{r}=4.5$ $\mathrm{cm}$ show the $\mathrm{q}=1$ island at $\sim 10 \mathrm{kHz}$ and harmonics. Fluctuations at $\mathrm{r}=10.3 \mathrm{~cm}$ exhibit nearly 'white' noise, lower than the preceding plot by two orders of magnitude or more, reaching a noise floor at $\sim 10^{-7}$. Fluctuation levels at $\mathrm{r}=10.3 \mathrm{~cm}$, measurable above the noise floor for $\mathrm{f}<20 \mathrm{kHz}$, are similar to or lower than the corresponding levels at $\mathrm{r}=4.5 \mathrm{~cm}$. 


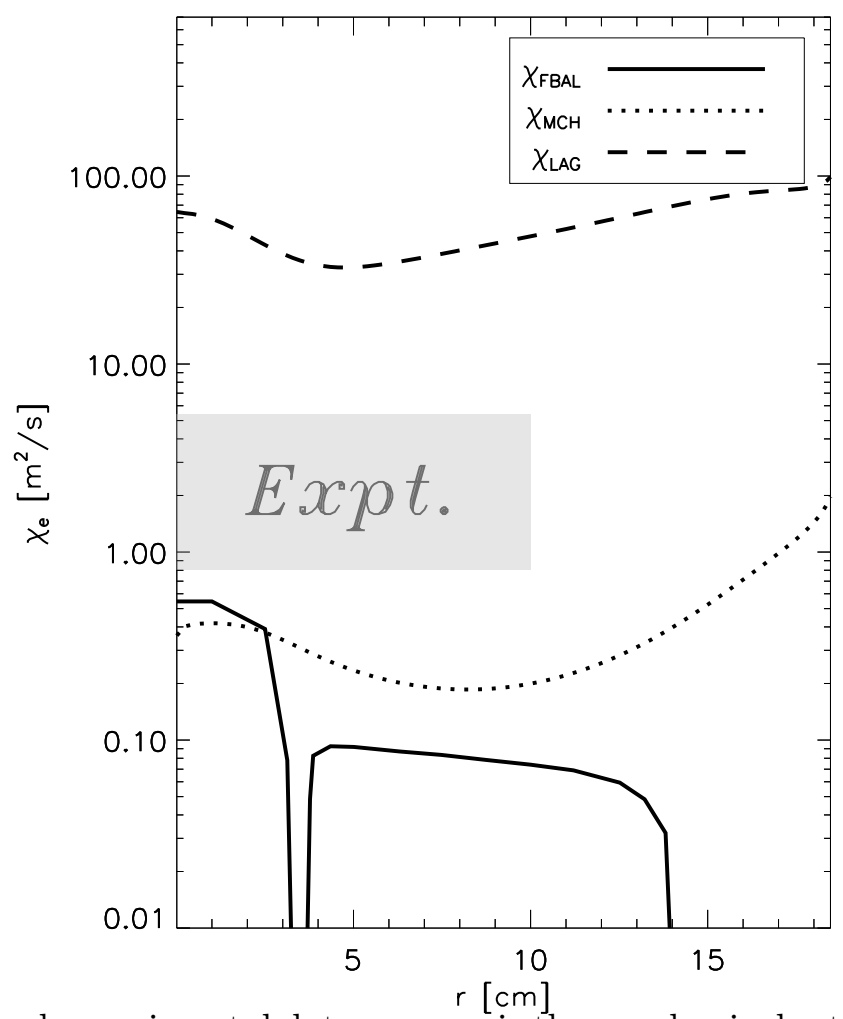

FIG. 6. $\chi_{\mathrm{e}}$ models and experimental data. $\chi_{\mathrm{FBAL}}$ is the neoclassical estimate from the FORCEBAL code, $\chi_{\mathrm{MCH}}$ is the modified Chang-Hinton estimate, and $\chi_{\mathrm{LAG}}$ is the Lackner-Gottardi model. The shaded region encompasses the range of $\chi_{\mathrm{e}}$ measurements from the temperature pulse experiments. The measurements also demonstrate sharply higher $\chi_{\mathrm{e}}$ for $\mathrm{r}>10 \mathrm{~cm}$. 


\section{ACKNOWLEDGMENTS}

The authors would like to acknowledge D. Hoffman, B. LeBlanc, and J. Taylor for their contribution to this research. This work was supported by US DOE contracts DE-AC0276CH03073 and DE-FG02-99ER54523. 


\section{REFERENCES}

[1] Y.-K. Peng and D. Strickler, Nucl. Fus. 26, 769 (1986).

[2] R. Stambaugh, V. Chan, R. Miller, and M. Schaffer, Fus. Technol. 33, 1 (1998).

[3] J. Menard et al., Nucl. Fus. 37, 595 (1997).

[4] R. Miller et al., Phys. Plasmas 4, 1062 (1997).

[5] T. Hender et al., in Plasma Physics and Controlled Nuclear Fusion Research 1992 (Proc. 14th Int. Conf. Wurzburg, 1992) Vol. 3, IAEA, Vienna 339 (1992).

[6] M. Gryaznevich et al., Phys. Rev. Lett. 80, 3972 (1998).

[7] D. Gates et al., Phys. Plasmas 5, 1775 (1998).

[8] G. Rewoldt, W. Tang, S. Kaye, and J. Menard, Phys. Plasmas 3, 1667 (1996).

[9] A. Sykes et al., Phys. Rev. Lett. 84, 495 (2000).

[10] Y.-K. Peng, Phys. Plasmas 7, 1681 (2000).

[11] R. Colchin et al., Phys. Fluids B 5, 2481 (1993).

[12] M. Walsh et al., in Proceedings of the 220nd European Physical Society Conference (PUBLISHER, Bournemouth, U.K., 1995), Vol. 3, p. 33.

[13] C. Roach, Plasma Phys. and Contr. Fus. 38, 2187 (1996).

[14] K. Lackner and N. Gottardi, Nucl. Fus. 30, 767 (1990).

[15] E. Fredrickson et al., Nucl. Fus. 26, 849 (1986).

[16] E. Fredrickson et al., Phys. Rev. Lett. 65, 2869 (1990).

[17] B. Tubbing, N. L. Cardozo, and M. V. D. Wiel, Nucl. Fus. 27, 1843 (1987).

[18] M. Kissick et al., Nucl. Fus. 34, 349 (1994). 
[19] K. Gentle et al., Physica Scripta 52, 411 (1995).

[20] H. Walter et al., Plasma Phys. and Contr. Fus. 40, 1661 (1998).

[21] L. Giannone et al., Nucl. Fus. 32, 1985 (1992).

[22] U. Gasparino et al., Plasma Phys. and Contr. Fus. 40, 233 (1998).

[23] P. Efthimion et al., Phys. Fluids B 3, 2315 (1991).

[24] E. Synakowski et al., Phys. Fluids B 5, 2215 (1993).

[25] N. L. Cardozo, Plasma Phys. and Contr. Fus. 37, 799 (1995).

[26] E. Fredrickson, M. Austin, and R. Groebner, Phys. Plasmas 7, 5051 (2000).

[27] D. Stutman et al., Rev. Sci. Instrum. 68, 1059 (1997), part II.

[28] M. Soler and J. Callen, Nucl. Fus. 19, 703 (1979).

[29] G. Taylor et al., Rev. Sci. Instrum. 72, 285 (2001).

[30] K. Gentle, Phys. Fluids 31, 1105 (1988).

[31] F. Levinton et al., Phys. Rev. Lett. 75, 4417 (1995).

[32] P. Efthimion et al., Nucl. Fus. 39, 1905 (1999).

[33] C. Greenfield et al., Nucl. Fus. 39, 1723 (1999).

[34] M. deBaar, M. Beurskens, G. Hogeweij, and N. Cardozo, Phys. Plasmas 6, 4645 (1999).

[35] S. Hirshman and D. Sigmar, Nucl. Fus. 21, 1079 (1981).

[36] C. Chang and F. Hinton, Phys. Fluids 29, 3314 (1986).

[37] W. Houlberg, K. Shaing, S. Hirshman, and M. Zarnstorff, Phys. Plasmas 4, 3230 (1997). 


\section{External Distribution}

Plasma Research Laboratory, Australian National University, Australia

Professor I.R. J ones, Flinders University, Australia

Professor J oão Canalle, Instituto de Fisica DEQ/IF - UERJ , Brazil

Mr. Gerson O. Ludwig, Instituto Nacional de Pesquisas, Brazil

Dr. P.H. Sakanaka, Instituto Fisica, Brazil

The Librarian, Culham Laboratory, England

Library, R61, Rutherford Appleton Laboratory, England

Mrs. S.A. Hutchinson, JET Library, England

Professor M.N. Bussac, Ecole Polytechnique, France

Librarian, Max-Planck-Institut für Plasmaphysik, Germany

J olan Moldvai, Reports Library, MTA KFKI-ATKI, Hungary

Dr. P. Kaw, Institute for Plasma Research, India

Ms. P.J . Pathak, Librarian, Insitute for Plasma Research, India

Ms. Clelia De Palo, Associazione EURATOM-ENEA, I taly

Dr. G. Grosso, Instituto di Fisica del Plasma, Italy

Librarian, Naka Fusion Research Establishment, J AERI, J apan

Library, Plasma Physics Laboratory, Kyoto University, J apan

Research Information Center, National Institute for Fusion Science, J apan

Dr. O. Mitarai, Kyushu Tokai University, J apan

Library, Academia Sinica, Institute of Plasma Physics, People's Republic of China

Shih-Tung Tsai, Institute of Physics, Chinese Academy of Sciences, People's Republic of China

Dr. S. Mirnov, TRINITI, Troitsk, Russian Federation, Russia

Dr. V.S. Strelkov, Kurchatov Institute, Russian Federation, Russia

Professor Peter Lukac, Katedra Fyziky Plazmy MFF UK, Mlynska dolina F-2, Komenskeho Univerzita, SK-842 15 Bratislava, Slovakia

Dr. G.S. Lee, Korea Basic Science Institute, South Korea

Mr. Dennis Bruggink, Fusion Library, University of Wisconsin, USA

Institute for Plasma Research, University of Maryland, USA

Librarian, Fusion Energy Division, Oak Ridge National Laboratory, USA

Librarian, Institute of Fusion Studies, University of Texas, USA

Librarian, Magnetic Fusion Program, Lawrence Livermore National Laboratory, USA

Library, General Atomics, USA

Plasma Physics Group, Fusion Energy Research Program, University of California at San Diego, USA

Plasma Physics Library, Columbia University, USA

Alkesh Punjabi, Center for Fusion Research and Training, Hampton University, USA

Dr. W.M. Stacey, Fusion Research Center, Georgia Institute of Technology, USA

Dr. J ohn Willis, U.S. Department of Energy, Office of Fusion Energy Sciences, USA

Mr. Paul H. Wright, Indianapolis, Indiana, USA 
The Princeton Plasma Physics Laboratory is operated by Princeton University under contract with the U.S. Department of Energy.

\author{
Information Services \\ Princeton Plasma Physics Laboratory \\ P.O. Box 451 \\ Princeton, NJ 08543
}

Phone: 609-243-2750

Fax: 609-243-2751

e-mail: pppl_info@pppl.gov

Internet Address: http://www.pppl.gov 